\title{
Cigarette smoke extract stimulates PCSK9 production in HepG2 cells via $\mathrm{ROS} / \mathrm{NF}-\kappa \mathrm{B}$ signaling
}

\author{
BAITAO MA ${ }^{1 *}$, XUEBIN WANG ${ }^{2 *}$, RUI ZHANG $^{1}$, SHUAI NIU $^{1}$, ZHIHUA RONG $^{1}$, \\ LENG NI $^{1}$, XIAO DI $^{1}, \mathrm{QIN} \mathrm{HAN}^{3}$ and CHANGWEI LIU ${ }^{1}$
}

\begin{abstract}
${ }^{1}$ Department of Vascular Surgery, Peking Union Medical College Hospital, Chinese Academy of Medical Sciences and Peking Union Medical College, Beijing 100730; ${ }^{2}$ Department of Vascular Surgery, Beijing Friendship Hospital, Capital Medical University, Beijing 100050; ${ }^{3}$ Center of Excellence in Tissue Engineering, Institute of Basic Medical Sciences and School of Basic Medicine, Chinese Academy of Medical Sciences and Peking Union Medical College, Beijing Key Laboratory of New Drug Development and Clinical Trial of Stem Cell Therapy, Beijing 100005, P.R. China
\end{abstract}

Received October 23, 2020; Accepted February 19, 2021

DOI: $10.3892 / \mathrm{mmr} .2021 .11970$

\begin{abstract}
Cigarette smoke (CS) exposure is a risk factor for dyslipidemia and atherosclerosis. Reduced expression of low-density lipoprotein receptor (LDLR) in hepatocytes may be one of the underlying mechanisms for these disorders. The aim of the present study was to investigate the molecular mechanism underlying the regulatory effect of CS extract (CSE) on proprotein convertase subtilisin/kexin type 9 (PCSK9) and low LDLR expression in HepG2 cells. PCSK9 and LDLR mRNA and protein expression levels in HepG2 cells were evaluated after CSE treatment via reverse transcription-quantitative polymerase chain reaction and western blotting, respectively. In addition, total intracellular reactive oxygen species (ROS) production was determined via 2,7-dichlorofluorescein diacetate fluorescence. CSE significantly increased PCSK9 expression and inhibited LDLR expression in a time- and concentration-dependent manner. Furthermore, CSE significantly induced ROS production and nuclear factor $\kappa \mathrm{B}(\mathrm{NF}-\kappa \mathrm{B})$ activation. However, pretreatment with a ROS scavenger or an NF- $\kappa \mathrm{B}$ inhibitor significantly attenuated the CSE-induced changes in PCSK9 and LDLR expression. In addition, pretreatment with melatonin markedly reduced ROS production, $\mathrm{NF}-\kappa \mathrm{B}$ activation and PCSK9 expression, and increased LDLR expression in the CSE-treated cells. These data suggest that melatonin inhibits CSE-regulated PCSK9 and LDLR production in HepG2 cells via ROS/NF- $\mathrm{B}$ signaling.
\end{abstract}

Correspondence to: Professor Changwei Liu, Department of Vascular Surgery, Peking Union Medical College Hospital, Chinese Academy of Medical Sciences and Peking Union Medical College, 1 Shuaifuyuan Hutong, Dongcheng, Beijing 100730, PR. China

E-mail: liucw@vip.sina.com

*Contributed equally

Key words: cigarette smoke extract, reactive oxygen species, $\mathrm{NF}-\kappa \mathrm{B}$, proprotein convertase subtilisin/kexin type 9 , low-density lipoprotein receptor, atherosclerosis

\section{Introduction}

Cigarette smoke (CS) exposure is one of the most important and modifiable risk factors for the development of atherosclerosis and associated cardiovascular and cerebrovascular diseases $(1,2)$, representing $31 \%$ of all global deaths in 2015 (3). Epidemiological studies of various populations have shown that CS exposure is a preventable risk factor for dyslipidemia (4-6). However, the cessation of cigarette smoking increases serum levels of high-density lipoprotein cholesterol (HDL-C) (5). In addition, exposure to CS for various time periods has been shown to cause dyslipidemia in various experimental animals (7-10). Dyslipidemia, particularly high levels of low-density lipoprotein cholesterol (LDL-C), is a key mechanism by which CS induces atherosclerosis (3). Previous studies have indicated that CS exposure raises total cholesterol and circulating LDL-C levels $(4,9,10)$. The accumulation of LDL-C in the subendothelial matrix is a primary event in atherosclerosis, and elevated levels of LDL-C in the circulation accelerate this process $(3,11)$. Our previous study demonstrated that CS exposure reduced the expression of the LDL receptor (LDLR) in mouse hepatocytes and HepG2 cells (10). However, the underlying mechanisms by which CS mediated the change in LDLR expression remain unclear.

Proprotein convertase subtilisin/kexin type 9 (PCSK9), which is mainly synthesized and secreted by hepatocytes, binds to the epidermal growth factor-like repeat homology domain of the LDLR on the surface of hepatocytes (12). The resulting complex is transported to the lysosome where it is degraded, increasing circulating LDL-C levels (12,13). PCSK9 loss-of-function mutations have been shown to be associated with low circulating levels of LDL-C and a reduced risk of coronary artery disease $(14,15)$. Monoclonal antibodies against PCSK9 have been used to treat patients with hyperlipidemia who are statin-intolerant or have familial hypercholesterolemia (16). In addition to increasing the circulating levels of LDL-C, PCSK9 plays an important role in inflammation (17). Lipopolysaccharide (LPS), tumor necrosis factor $\alpha(\mathrm{TNF}-\alpha)$ and reactive oxygen species (ROS) markedly increase the expression of PCSK9 in vivo and in vitro (17). However, the effect of CS on PCSK9 expression has not yet been elucidated. 
Melatonin has a variety of physiological functions, including a regulatory effect of circadian rhythm, as well as anti-inflammatory and antioxidant activity (18). Our previous study showed that melatonin increased the expression of LDLR in mouse hepatocytes and HepG2 cells (10). Other studies have demonstrated that melatonin exerts anti-inflammatory and antioxidant effects via the upregulation of sirtuin 1 (SIRT1) activity and expression (19-21). However, whether melatonin and SIRT1 mediate the CSE-induced regulation of PCSK9 and LDLR expression remains unclear.

We hypothesize that CS and PCSK9/LDLR expression may be linked. To examine this postulation, HepG2 cells were treated with CS extract (CSE) and then the mRNA and protein expression levels of PCSK9 and LDLR were evaluated via reverse transcription-quantitative polymerase chain reaction (RT-qPCR) and western blotting, respectively. In addition, the role of ROS/nuclear factor $\kappa \mathrm{B}(\mathrm{NF}-\mathrm{\kappa B})$ signaling in the regulation of PCSK9 and LDLR expression by CSE was studied.

\section{Materials and methods}

Cell culture and treatment. HepG2 cells were purchased from the American Type Culture Collection, and cultured in Dulbecco's modified Eagle's medium (DMEM, HyClone; Cytiva) supplemented with $10 \%$ fetal bovine serum (FBS; HyClone; Cytiva) and $1 \%(\mathrm{v} / \mathrm{v})$ penicillin/streptomycin at $37^{\circ} \mathrm{C}$ with $5 \% \mathrm{CO}_{2}$. The cells were stimulated with $0,1.25,2.5$ and $5 \% \mathrm{CSE}$ for $24 \mathrm{~h}$ or with $5 \% \mathrm{CSE}$ for 6,12 and $24 \mathrm{~h}$ at $37^{\circ} \mathrm{C}$. The concentrations of CSE were selected according to our previous study (10). The medium was changed to DMEM containing $1 \%$ FBS during treatment with CSE and/or $5 \mathrm{mM} \mathrm{ROS} \mathrm{inhibitor}$ [ $N$-acetyl-L-cysteine (NAC); Sigma-Aldrich; Merck KGaA], $10 \mu \mathrm{M}$ NF- $\kappa \mathrm{B}$ inhibitor (BAY11-7082; Sigma-Aldrich, Merck $\mathrm{KGaA}), 100 \mu \mathrm{M}$ melatonin (Sigma-Aldrich; Merck KGaA) and $2 \mu \mathrm{M}$ SIRT1 inhibitor (Inauhzin; Sigma-Aldrich; Merck $\mathrm{KGaA}$ ). In most experiments, HepG2 cells were pretreated with or without BAY11-7082, melatonin or Inauhzin for $1 \mathrm{~h}$ prior to stimulation with $5 \% \mathrm{CSE}$ for $24 \mathrm{~h}$ at $37^{\circ} \mathrm{C}$. However, the expression level of phosphorylated protein was measured after treatment with CSE for only $30 \mathrm{~min}$, as it would be degraded if the treatment was prolonged. All experiments were performed at least three times and representative results are shown.

Preparation of CSE. CSE was obtained from ordinary filtered cigarettes (each containing $0.90 \mathrm{mg}$ nicotine and $10.0 \mathrm{mg}$ tar) for all experiments. One cigarette was bubbled into $15 \mathrm{ml}$ DMEM, which was then adjusted to $\mathrm{pH} 7.4$ and filtered through a $0.22-\mathrm{mm}$ filter (Roche Diagnostics). This solution was defined as 100\% CSE. The CSE used in all experiments was freshly prepared.

Western blot analysis. Total protein from HepG2 cells was prepared using lysis buffer $(50 \mathrm{mM}$ Tris, $150 \mathrm{mM} \mathrm{NaCl}$, $1 \%$ NP-40, $0.5 \%$ sodium deoxycholate, $0.1 \%$ SDS; pH 7.4) with $1 \mathrm{mM}$ phenylmethylsulfonyl fluoride (Beyotime Institute of Biotechnology) on ice; when detecting phosphorylated protein, $1 \%(\mathrm{v} / \mathrm{v})$ phosphatase inhibitor (Shanghai Yeasen Biotechnology Co., Ltd.) was added to the lysis solution. The lysates were centrifuged at $13,000 \mathrm{x}$ g for $20 \mathrm{~min}$ at $4^{\circ} \mathrm{C}$ to remove cell debris, and the total protein concentra- tion was determined using a bicinchoninic acid protein assay kit (Beyotime Institute of Biotechnology) according to the manufacturer's instructions. Protein ( $20 \mu \mathrm{g} / \mathrm{lane})$ was separated by $4-15 \%$ sodium dodecyl sulfate-polyacrylamide gel electrophoresis (Applygen Technologies, Inc.) and then electroblotted onto polyvinylidene fluoride membranes. After blocking with 5\% skimmed milk or 3\% bovine serum albumin (Shanghai Yeasen Biotechnology Co., Ltd.) in Tris-buffered saline containing $0.1 \%$ Tween-20 (TBST) for $60 \mathrm{~min}$ at room temperature, the membranes were incubated with primary antibodies against the following: PCSK9 (1:1,000; cat. no. 85813S; Cell Signaling Technology, Inc.), LDLR (1:1,000; cat. no. 10785-1-AP; ProteinTech Group, Inc.), phosphorylated (p)-p65 (1:1,000; cat. no. sc-136548; Santa Cruz Biotechnology, Inc.), total p65 (1:1,000; cat. no. sc-8008; Santa Cruz Biotechnology, Inc.) and glyceraldehyde-3-phosphate dehydrogenase (GAPDH; 1:10,000; cat. no. 10494-1-AP; ProteinTech Group, Ltd.) overnight at $4^{\circ} \mathrm{C}$ in a shaker. Then, the membranes were washed with TBST (three times for $10 \mathrm{~min}$ each) before incubating with the secondary anti-rabbit horseradish peroxidase-conjugated antibody $(1: 5,000$; cat. no. ZB-2301; Beijing Zhongshan Jinqiao Biotechnology Co., Ltd.) for $60 \mathrm{~min}$ at room temperature. The antibody-antigen complexes were detected using enhanced electrochemiluminescence reagents (Shanghai Yeasen Biotechnology Co., Ltd.) and densitometrically analyzed with ImageJ software (version 1.46; National Institutes of Health).

$R T-q P C R$. Total RNA was extracted from cells using TRIzol ${ }^{\circledR}$ (Invitrogen; Thermo Fisher Scientific, Inc.) and quantified with a NanoDrop ${ }^{\mathrm{TM}} 2000$ Spectrophotometer (Thermo Fisher Scientific, Inc.). Complementary DNA (cDNA) was synthesized with $1 \mu \mathrm{g}$ total RNA using a High-Capacity cDNA Reverse Transcription Kit (Takara Bio, Inc.), the temperature protocol was as follows: $42^{\circ} \mathrm{C}$ for $60 \mathrm{~min}, 70^{\circ} \mathrm{C}$ for $15 \mathrm{~min}$, and then $4^{\circ} \mathrm{CC}$ for $5 \mathrm{~min}$. qPCR was then performed in a CFX96 Touch Real-Time PCR Detection System (Bio-Rad Laboratories, Inc.) using SYBR Premix (Shanghai Yeasen Biotechnology Co., Ltd.), the thermocycling conditions were as follows: Predenaturation step at $95^{\circ} \mathrm{C}$ for $3 \mathrm{~min}$, followed by 40 cycles of $95^{\circ} \mathrm{C}$ for $10 \mathrm{sec}$ and $60^{\circ} \mathrm{C}$ for $40 \mathrm{sec}$. Each sample was performed in triplicate. Table I lists the primer sequences used. The relative mRNA expression levels were normalized to $G A P D H$ expression using the $2^{-\triangle \Delta C q}$ method (22).

Measurement of ROS production. Total intracellular ROS generation was measured using an ROS Assay Kit (Beyotime Institute of Biotechnology) according to the manufacturer's instructions. HepG2 cells were pretreated with NAC $(5 \mathrm{mM})$, BAY11-7082 $(10 \mu \mathrm{M})$ or melatonin $(100 \mu \mathrm{M})$ for $1 \mathrm{~h}$, and then stimulated with $5 \% \mathrm{CSE}$ for $24 \mathrm{~h}$ at $37^{\circ} \mathrm{C}$. After that, the medium was replaced with serum-free culture medium containing $10 \mu \mathrm{M}$ 2,7-dichlorodihydrofluorescein diacetate (DCFH-DA) for $20 \mathrm{~min}$ at $37^{\circ} \mathrm{C}$, and then the cells were washed three times with serum-free medium. The green fluorescence was measured using a fluorescence microscope using an excitation of $488 \mathrm{~nm}$ and an emission of $525 \mathrm{~nm}$.

Statistical analysis. All data are presented as the mean \pm standard deviation of three different experiments in triplicate. 
Table I. Sequences of primers.

\begin{tabular}{lll}
\hline Gene & \multicolumn{1}{c}{ Forward $\left(5^{\prime} \rightarrow 3^{\prime}\right)$} & \multicolumn{1}{c}{ Reverse $\left(5^{\prime} \rightarrow 3^{\prime}\right)$} \\
\hline PCSK9 & CCTGCGCGTGTCAACT & GCTGGCTTTTCCGAAACTC \\
LDLR & GTGTCACAGCGGCG & CGCACTCTTTGATG \\
GAPDH & GAAGGTGAAGGTCGGAGTC & GAAGATGGTGATGGGATTTC
\end{tabular}

PCSK9, proprotein convertase subtilisin/kexin type 9; LDLR, low-density lipoprotein receptor; GAPDH, glyceraldehyde-3-phosphate dehydrogenase.
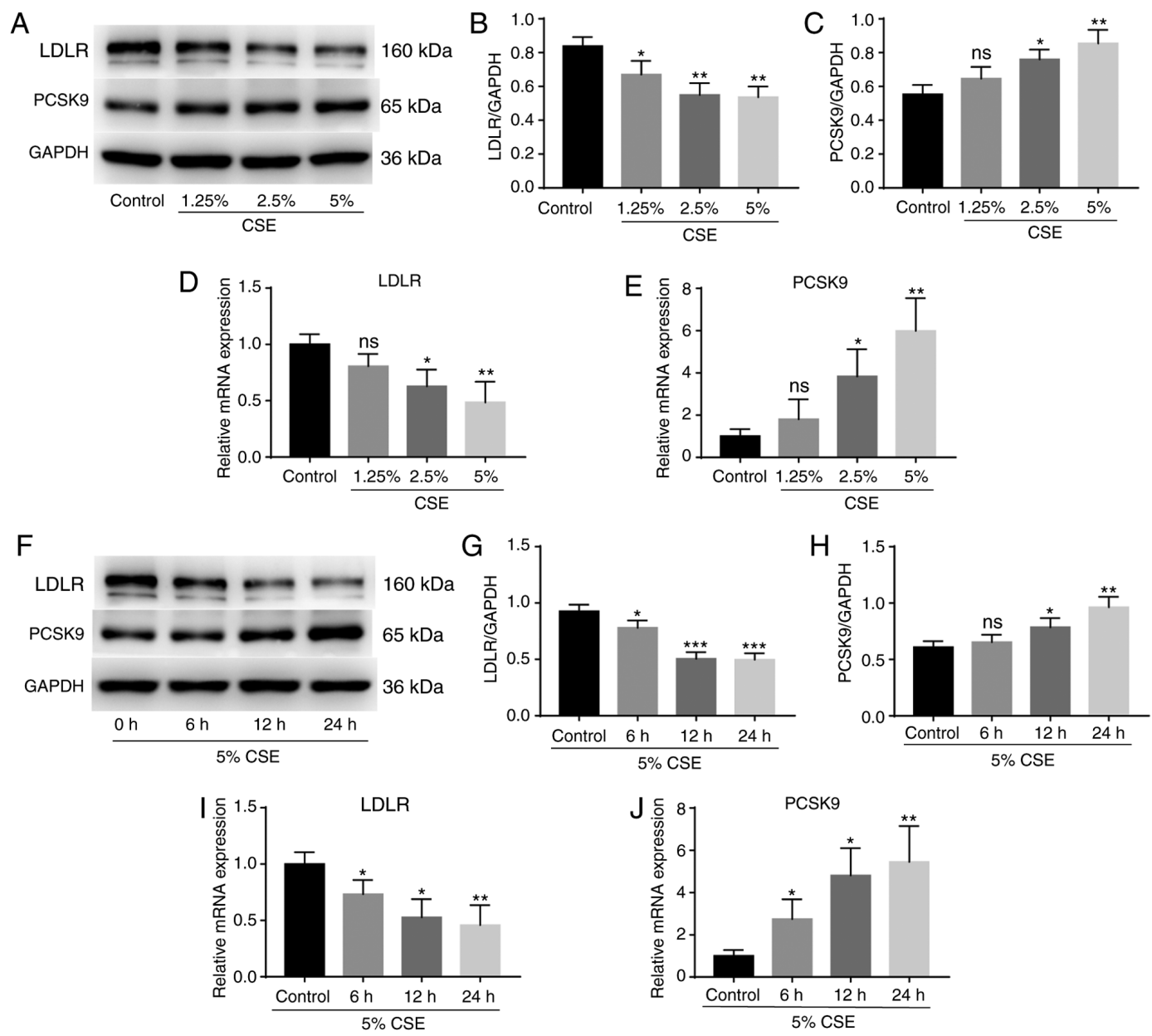

Figure 1. Effects of CSE on PCSK9 and LDLR production in HepG2 cells. (A) Western blot analysis of PCSK9 and LDLR expression in HepG2 cells treated with 0-5\% CSE for $24 \mathrm{~h}$. Semi-quantitative analysis of (B) LDLR and (C) PCSK9 relative expression. RT-qPCR analysis of (D) LDLR and (E) PCSK9 mRNA expression. (F) Western blot analysis of PCSK9 and LDLR expression in HepG2 cells treated with 5\% CSE for 6, 12 and 24 h. Semi-quantitative analysis of (G) LDLR and (H) PCSK9 relative expression. RT-qPCR analysis of (I) LDLR and (J) PCSK9 mRNA expression. ${ }^{*} \mathrm{P}<0.05$, ${ }^{* * *} \mathrm{P}<0.01$ and ${ }^{* * * *} \mathrm{P}<0.001 \mathrm{vs}$. control group; ns, not significant $(\mathrm{P}>0.05)$. CSE, cigarette smoke extract; PCSK9, proprotein convertase subtilisin/kexin type 9; LDLR, low-density lipoprotein receptor; GAPDH, glyceraldehyde-3-phosphate dehydrogenase; RT-qPCR, reverse transcription-quantitative polymerase chain reaction.

The data were analyzed using one-way analysis of variance with Bonferroni test (GraphPad Prism Version 7.0; GraphPad Software, Inc.). All data were obtained from at least three independent experiments. $\mathrm{P}<0.05$ was considered to indicate a statistically significant result.

\section{Results}

Effects of CSE on PCSK9 and LDLR expression in HepG2 cells. To investigate whether CSE regulates PCSK9 and LDLR expression, HepG2 cells were treated with 0, 1.25, 2.5 and $5 \% \mathrm{CSE}$ for $24 \mathrm{~h}$, and the expression levels of PCSK9 and LDLR were determined using western blotting and RT-qPCR. CSE reduced the expression of LDLR protein (Fig. 1A and B) and mRNA (Fig. 1D) and induced the expression of PCSK9 protein (Fig. 1A and C) and mRNA (Fig. 1E) in a concentration-dependent manner. Then, HepG2 cells were stimulated with 5\% CSE for 6, 12 and $24 \mathrm{~h}$. The results revealed that CSE decreased the production of LDLR protein (Fig. 1F and G) and mRNA (Fig. 1I) and induced the production of PCSK9 protein 
A

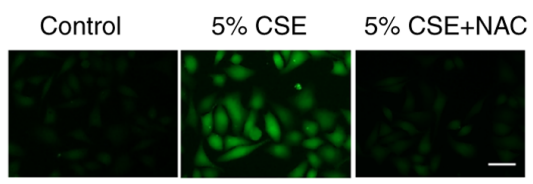

B

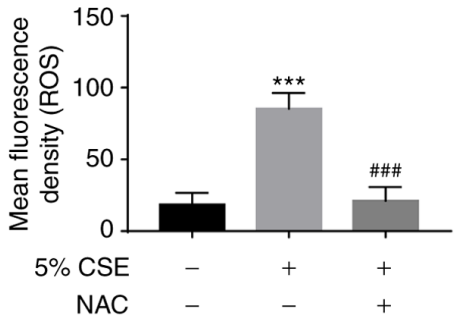

C

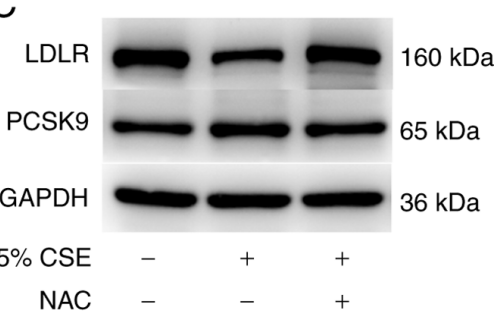

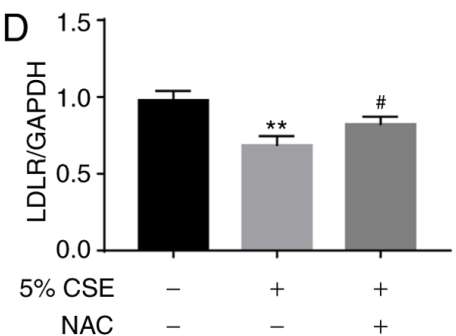

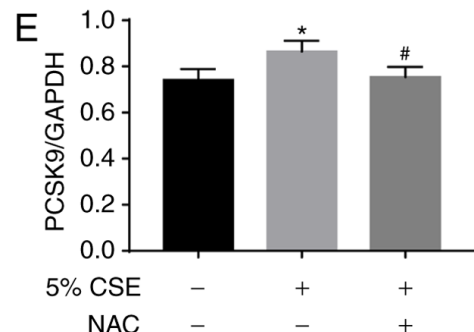

Figure 2. Role of ROS in PCSK9 and LDLR expression in CSE-stimulated HepG2 cells. (A) Total ROS levels were measured using 2,7-dichlorodihydrofluorescein diacetate $(10 \mu \mathrm{M})$ in HepG2 cells with or without $5 \mathrm{mM} \mathrm{NAC}$ treatment for $1 \mathrm{~h}$ prior to treatment with 5\% CSE for $24 \mathrm{~h}$. Scale bar, $50 \mu \mathrm{m}$. (B) Mean fluorescence density of ROS. (C) Western blot analysis of LDLR and PCSK9 expression. Semi-quantitative analysis of (D) LDLR and (E) PCSK9 relative expression. ${ }^{*} \mathrm{P}<0.05,{ }^{* *} \mathrm{P}<0.01$ and ${ }^{* * * *} \mathrm{P}<0.001$ vs. control group. ${ }^{\#} \mathrm{P}<0.05$ and ${ }^{\# \# \#} \mathrm{P}<0.001$ vs. CSE group. ROS, reactive oxygen species; CSE, cigarette smoke extract; PCSK9, proprotein convertase subtilisin/kexin type 9; LDLR, low-density lipoprotein receptor; GAPDH, glyceraldehyde-3-phosphate dehydrogenase; NAC, N-acetyl-L-cysteine.
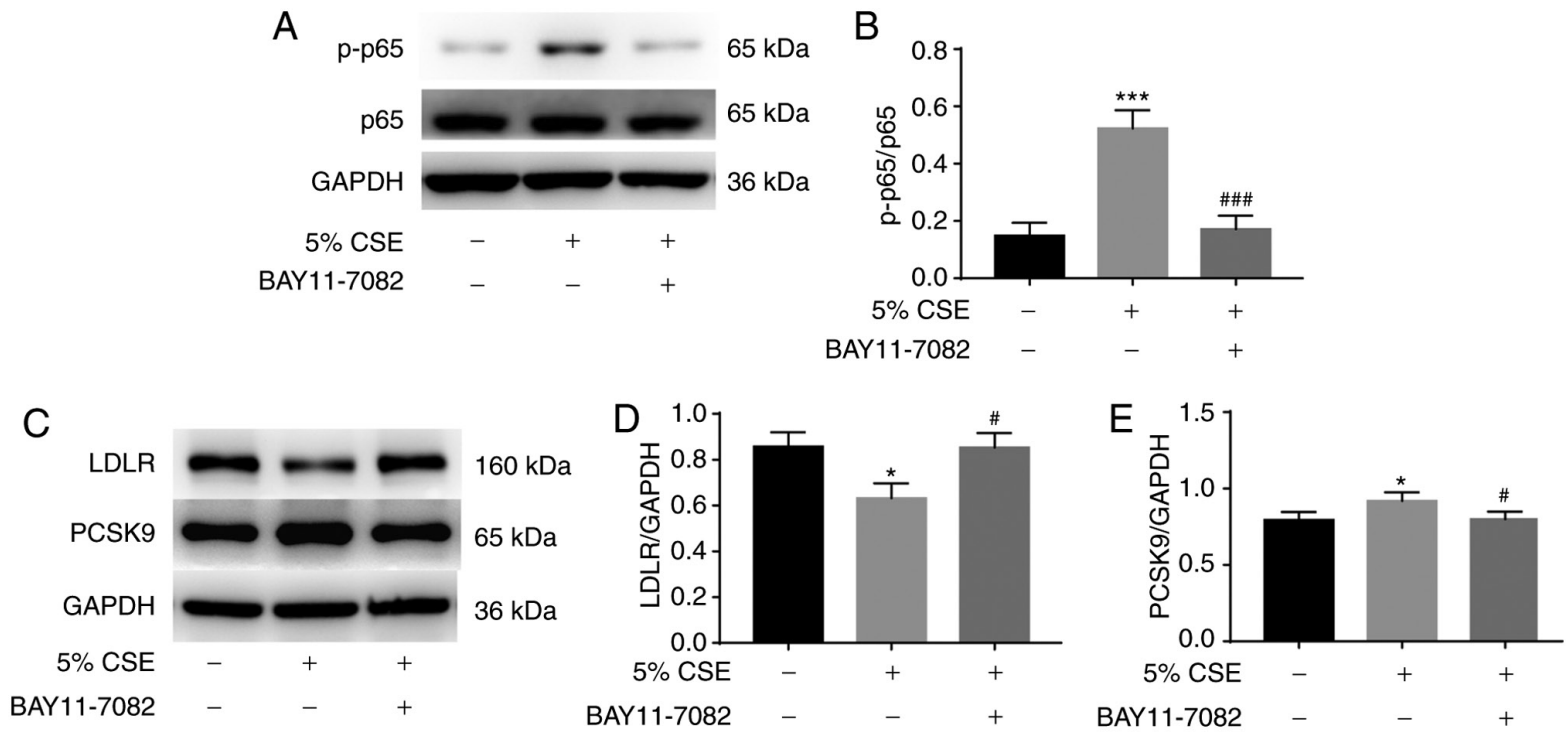

Figure 3. Effects of NF-kB activation on PCSK9 and LDLR expression in CSE-stimulated HepG2 cells. HepG2 cells were treated with or without $10 \mu \mathrm{M}$ BAY11-7082 for $1 \mathrm{~h}$ prior to stimulation with 5\% CSE for $30 \mathrm{~min}$. (A) Western blot analysis of p-p65 and total p65 to measure NF- $\mathrm{kB}$ activation. (B) Semi-quantitative analysis of the p-p65/p65 ratio. (C) LDLR and PCSK9 expression levels in HepG2 cells after stimulation with 5\% CSE for $24 \mathrm{~h}$, with or without BAY11-7082 pretreatment. Semi-quantitative analysis of (D) LDLR and (E) PCSK9 relative expression. ${ }^{*} \mathrm{P}<0.05$ and ${ }^{* * * *} \mathrm{P}<0.001$ vs. control group. ${ }^{\#} \mathrm{P}<0.05$ and ${ }^{\# \# \#} \mathrm{P}<0.001$ vs. CSE group. CSE, cigarette smoke extract; PCSK9, proprotein convertase subtilisin/kexin type 9; LDLR, low-density lipoprotein

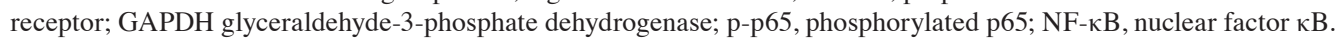

(Fig. 1F and $\mathrm{H}$ ) and mRNA (Fig. 1J) in a time-dependent manner.

Effects of ROS on PCSK9 and LDLR expression in CSE-stimulated HepG2 cells. To investigate whether ROS contribute to the effect of CSE on PCSK9 and LDLR expression, HepG2 cells were incubated with or without $5 \mathrm{mM}$ NAC for $1 \mathrm{~h}$ prior to stimulation with $5 \% \mathrm{CSE}$ for $24 \mathrm{~h}$, and then total intracellular ROS generation was measured using DCFH-DA. CSE treatment significantly increased ROS production; however, pretreatment with NAC significantly inhibited the
CSE-induced production of ROS (Fig. 2A and B). In addition, the effects of NAC on LDLR and PCSK9 expression were measured using western blotting. The pretreatment of HepG2 cells with NAC attenuated the inhibitory effect of CSE on LDLR expression (Fig. 2C and D) and the stimulatory effect of CSE on PCSK9 expression (Fig. 2C and E).

Effects of $N F-\kappa B$ activation on PCSK9 and LDLR expression in CSE-stimulated Hep 22 cells. Next, whether NF- $\kappa \mathrm{B}$ activation contributes to the effect of CSE on PCSK9 and LDLR expression was investigated. HepG2 cells were incubated with 

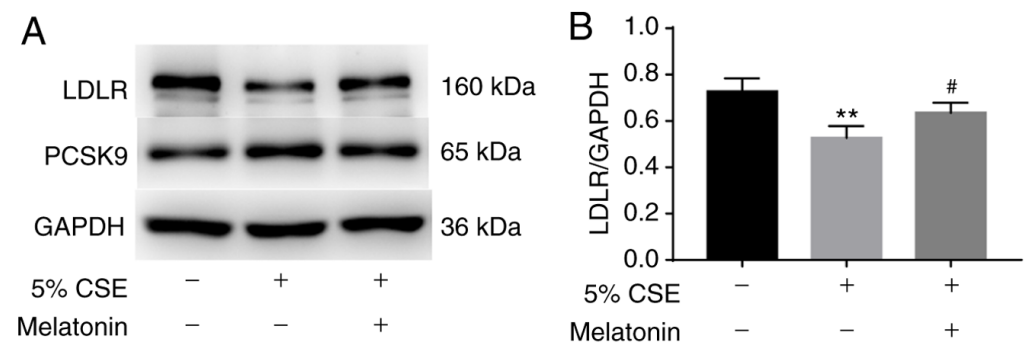
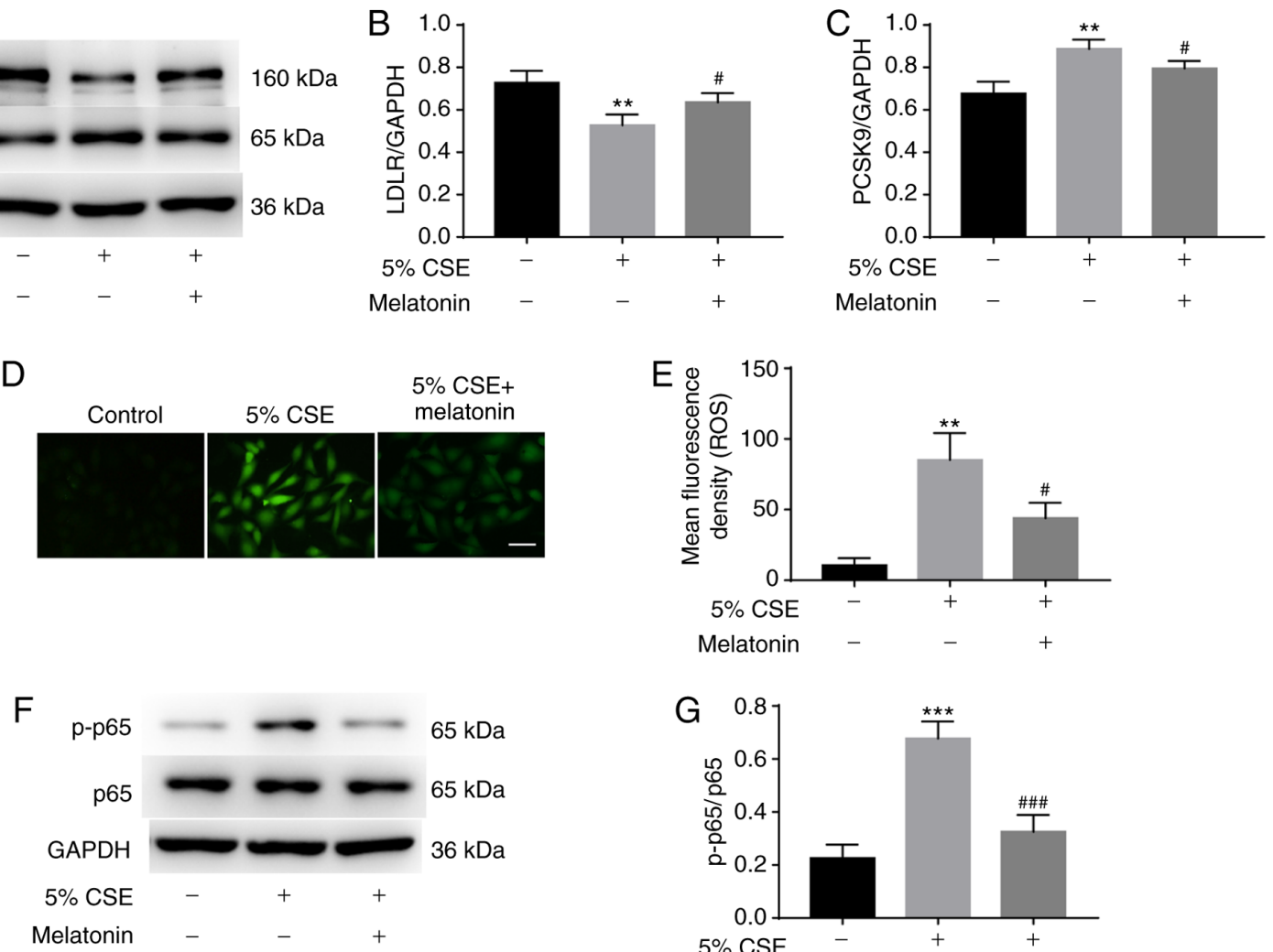

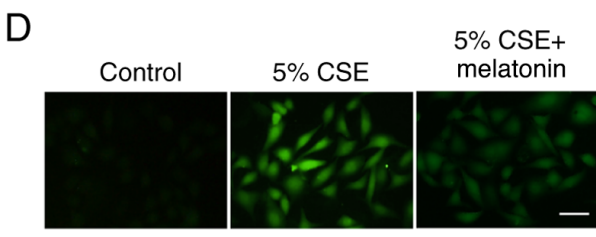

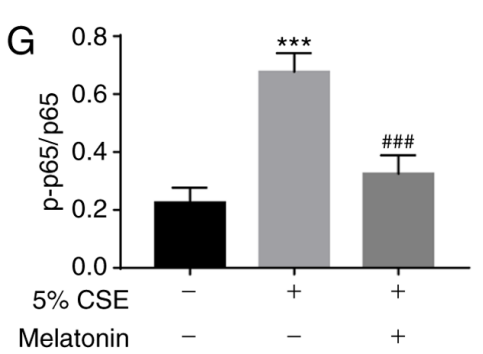

Figure 4. Effects of melatonin on CSE-regulated PCSK9 and LDLR expression in HepG2 cells. HepG2 cells were treated with or without $100 \mu$ M melatonin for $1 \mathrm{~h}$ prior to stimulation with 5\% CSE for $24 \mathrm{~h}$. (A) Western blot analysis of LDLR and PCSK9 expression. Semi-quantitative analysis of (B) LDLR and (C) PCSK9 relative expression. (D) Total ROS levels were measured using $10 \mu \mathrm{M}$ 2,7-dichlorodihydrofluorescein diacetate. Scale bar, 50 $\mu$ m. (E) Mean fluorescence density of ROS. (F) Western blot analysis of p-p65 and total p65 in HepG2 cells after stimulation with 5\% CSE for 30 min. (G) Semi-quantitative analysis of the p-p65/p65 ratio. ${ }^{* *} \mathrm{P}<0.01$ and ${ }^{* * *} \mathrm{P}<0.001$ vs. control group. ${ }^{\#} \mathrm{P}<0.05$ and ${ }^{\# \# \#} \mathrm{P}<0.001$ vs. CSE group. CSE, cigarette smoke extract; PCSK9, proprotein convertase subtilisin/kexin type 9; LDLR, low-density lipoprotein receptor; GAPDH, glyceraldehyde-3-phosphate dehydrogenase; ROS, reactive oxygen species; p-p65, phosphorylated p65.
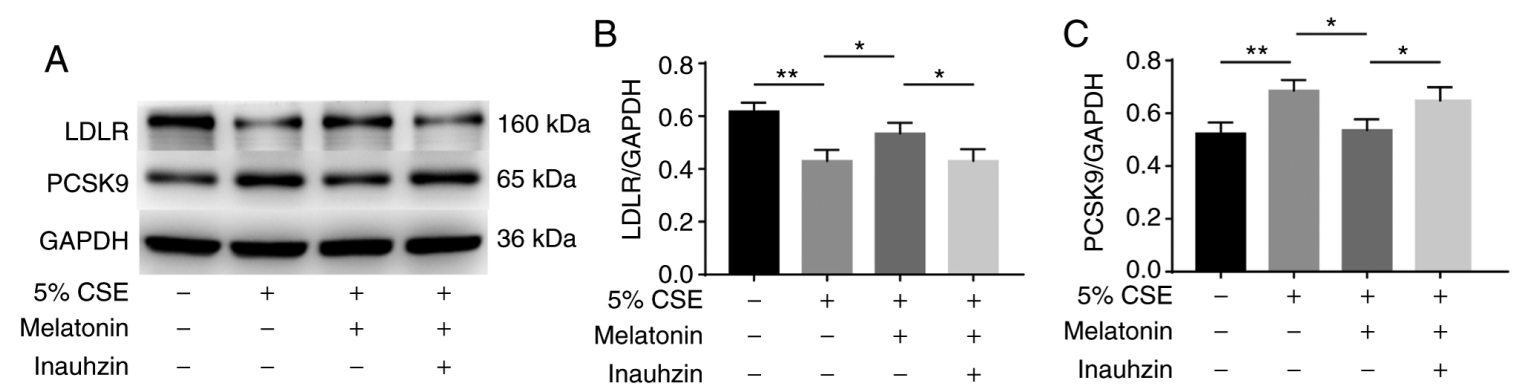

Figure 5. Role of SIRT1 in the modulatory effect of melatonin on CSE-regulated PCSK9 and LDLR expression in HepG2 cells. HepG2 cells were untreated or treated with $100 \mu \mathrm{M}$ melatonin alone or with $2 \mu \mathrm{M}$ Inauhzin for $1 \mathrm{~h}$, prior to treatment with 5\% CSE for $24 \mathrm{~h}$. (A) Western blot analysis of LDLR and PCSK9 expression levels. Semi-quantitative analysis of (B) LDLR and (C) PCSK9 relative expression. ${ }^{*} \mathrm{P}<0.05$ and ${ }^{* *} \mathrm{P}<0.01$. CSE, cigarette smoke extract; PCSK9, proprotein convertase subtilisin/kexin type 9; LDLR, low-density lipoprotein receptor; GAPDH, glyceraldehyde-3-phosphate dehydrogenase; SIRT1, sirtuin 1.

or without $10 \mu \mathrm{M}$ BAY11-7082 for $1 \mathrm{~h}$, and then stimulated with 5\% CSE. Stimulation with 5\% CSE for $30 \mathrm{~min}$ induced the phosphorylation of p65 significantly; however, this increase was attenuated by BAY11-7082 pretreatment (Fig. 3A and B). Furthermore, BAY11-7082 pretreatment also reversed the inhibition of LDLR expression and increase in PCSK9 expression induced by treatment with $5 \%$ for $24 \mathrm{~h}$ (Fig. 3C-E).

Effects of melatonin on CSE-regulated PCSK9 and LDLR production. To assess whether melatonin inhibited
CSE-regulated PCSK9 and LDLR production, HepG2 cells were pretreated with or without $100 \mu \mathrm{M}$ melatonin for $1 \mathrm{~h}$ and then stimulated with 5\% CSE for $24 \mathrm{~h}$. The inhibitory effect of CSE treatment on LDLR expression was reversed by melatonin, and the CSE-induced increase in PCSK9 expression was also attenuated by melatonin (Fig. 4A-C).

Whether ROS and/or NF- $\mathrm{KB}$ are involved in the mechanism by which melatonin regulates CSE-induced changes in PCSK9 and LDLR expression was then examined. The levels of ROS were measured using DCFH-DA, and p-p65 
levels were measured by western blotting. Pretreatment with melatonin significantly decreased the production of ROS (Fig. 4D and E) and the phosphorylation of p65 (Fig. 4F and G) in the CSE-stimulated HepG2 cells.

Effects of SIRT1 on the regulation of PCSK9 and LDLR expression by melatonin in CSE-stimulated HepG2 cells. The role of SIRT1 in the regulatory effect of melatonin on PCSK9 and LDLR production was also investigated in CSE-stimulated cells. HepG2 cells were treated with $100 \mu \mathrm{M}$ melatonin alone or with $2 \mu \mathrm{M}$ Inauhzin for $1 \mathrm{~h}$, and then stimulated with $5 \% \mathrm{CSE}$ for $24 \mathrm{~h}$. The CSE-induced reduction in LDLR expression was attenuated by melatonin, and Inauhzin blocked the effect of melatonin (Fig. 5A and B). In addition, the CSE-induced increase in PCSK9 expression was suppressed by melatonin, and Inauhzin blocked the inhibitory effect of melatonin (Fig. 5A and C).

\section{Discussion}

The present study demonstrated that CSE induced PCSK9 expression and decreased LDLR expression in HepG2 cells, while melatonin attenuated those effects. Furthermore, CSE induced ROS production and NF- $\mathrm{NB}$ activation, and pretreatment with a ROS scavenger or $\mathrm{NF}-\kappa \mathrm{B}$ inhibitor suppressed the CSE-mediated regulation of PCSK9 and LDLR expression in the cells. These data indicate that CSE induced PCSK9 expression and inhibited LDLR expression via ROS and NF- $\kappa \mathrm{B}$ signaling pathways in the HepG2 cells.

Epidemiological studies have shown that dyslipidemia is associated with CS exposure (23-26); smokers have higher serum levels of cholesterol (24), higher plasma triglyceride concentrations (25) and lower concentrations of HDL-C (26) than non-smokers, and smoking cessation for $>6$ years can reduce the risk of dyslipidemia (23). Animal experiments have also shown that CS exposure can cause dyslipidemia $(7,27)$. LDL-C is an indubitable causal factor in atherosclerosis $(3,28)$. Long-term exposure to CS has been shown to increase serum LDL-C levels in experimental animals (9), and our previous study (10) indicated that the reduced expression of the LDLR on hepatocytes caused by CS exposure may underlie the increase in serum LDL-C levels. The present study demonstrated that CSE stimulated the expression of PCSK9 mRNA and protein, which may explain the mechanism by which CSE inhibits LDLR expression.

PCSK9 serves a critical role in the regulation of cholesterol homeostasis $(13,29)$. It binds the LDLR at the surface of hepatocytes, activating the endosomal and lysosomal degradation of LDLR in the liver, resulting in increased serum LDL-C levels $(30,31)$. In the present study, CSE induced PCSK9 expression in HepG2 cells in a time- and concentration-dependent manner, consistent with the decreased LDLR expression. However, in addition to decreasing LDLR at the surface of hepatocytes, another important role of PCSK9 is the regulation of inflammation (17). Pro-inflammatory factors such as LPS, TNF- $\alpha$ and oxidized LDL (ox-LDL) have been shown to upregulate PCSK9 expression (17). Moreover, compared with wild-type mice, PCSK9 knockout mice display a decreased response to LPS stimulation, manifested by the reduced production of inflammatory factors such as TNF- $\alpha$, interleukin
(IL)-6, monocyte chemotactic protein 1 and IL-1 $\beta(32,33)$, which suggests that PCSK9 may participate in the process of inflammation. The present study demonstrated that CSE induced PCSK9 expression in HepG2 cells, and suggests that PCSK9 may play a role in CSE-induced inflammation.

CSE simulates the various harmful substances contained in real CS, including high concentrations of oxidants, which can induce ROS production and are important in the pathogenesis of atherosclerosis $(1,2)$. In our previous study, we reported that CSE-induced pyroptosis in human umbilical vein endothelial cells (ECs) required ROS, and that CS increased the production of ROS in rat carotid arteries (34). Notably, Ding et al (35) reported that hemodynamic shear stress modulated PCSK9 expression in human primary aortic ECs and smooth muscle cells (SMCs) via ROS production. Furthermore, another study demonstrated PCSK9 expression was enhanced by the induction of mitochondrial ROS and reduced by their inhibition (33). In the present study, the increased PCSK9 expression induced by CSE was inhibited by NAC, a ROS scavenger, which indicates that CSE induced PCSK9 expression via ROS.

The transcription factor $\mathrm{NF}-\kappa \mathrm{B}$ is known as the master regulator of inflammation and immune homeostasis (36), and CS can induce its activation (37). Inhibiting the activity of $N F-\kappa B$ has been shown to alleviate the progression of atherosclerosis $(38,39)$. By contrast, the autophagy of SMCs induced by nicotine, one of the main components in $\mathrm{CS}$, accelerates atherosclerosis via ROS/NF- $\kappa$ B signaling (40). Furthermore, LPS, ox-LDL and TNF- $\alpha$ have been demonstrated to regulate PCSK9 expression via the NF- $\mathrm{B}$ signaling pathway (33). NF- $\kappa \mathrm{B}$ downstream signaling, including IL-1 $\beta$, IL-6 and TNF- $\alpha$, has also been shown to significantly induce PCSK9 expression (41). The present study demonstrated that the inhibition of $\mathrm{NF}-\kappa \mathrm{B}$ activation significantly suppressed CSE-induced PCSK9 expression, suggesting that CSE induced PCSK9 expression via $N F-\kappa B$ signaling. Notably, there is complex crosstalk between ROS and the NF- $\kappa \mathrm{B}$ signaling pathway; $N F-\kappa B$ regulatory genes are important in regulating the amount of ROS in cells, and ROS have inhibitory or stimulatory effects on NF- $\mathrm{NB}$ signal transduction (42). However, this relationship was not investigated further as it was not the focus of the present study.

A meta-analysis of 12 randomized controlled trials found that melatonin ameliorates dyslipidemia and reduces LDL-C and triglyceride levels (43). In addition, studies have shown that treatment with melatonin regulates dyslipidemia in rats (44-46) and can reprogram the gut microbiota to improve lipid dysmetabolism in mice fed a high-fat diet (47). In the present study, melatonin attenuated the CSE-induced increase in PCSK9 expression and reduction in LDLR expression, which indicates that melatonin regulated CSE-induced dyslipidemia by downregulating PCSK9 expression and upregulating LDLR expression. In addition, the present study demonstrated that melatonin significantly decreased ROS production and p65 phosphorylation in CSE-stimulated HepG2 cells, suggesting that melatonin regulated PCSK9 and LDLR expression by blocking the ROS and NF- $\mathrm{BB}$ signaling pathways in HepG2 cells.

Multiple studies have shown that melatonin exerts anti-inflammatory and antioxidant effects by upregulating SIRT1 activity and expression (19-21), and that SIRT1 activation 
inhibits NF- $\kappa \mathrm{B}$ signaling $(48,49)$, thereby protecting the cells from ROS (50). The results of the present study indicate that the inhibition of SIRT1 reversed the regulatory effect of melatonin on CSE-induced PCSK9 and LDLR expression. The absence of in vivo data to confirm the results is a potential limitation of the present study, and such experiments are planned in the future.

In conclusion, the present study showed that CSE increased PCSK9 production and inhibited LDLR expression in HepG2 cells. Additionally, it demonstrated that ROS and NF- $\kappa \mathrm{B}$ signaling pathways are mediators of CSE-regulated PCSK9 and LDLR expression, which may contribute to the lipid metabolism disorders caused by CS. Melatonin regulated PCSK 9 and LDLR expression via SIRT1, which blocked the ROS/NF- $\mathrm{B}$ signaling in HepG2 cells. However, additional experiments are required to clarify the results.

\section{Acknowledgements}

The authors would like to thank Professor Jing Li and Professor Shihua Wang of the Medical Science Research Center, Chinese Academy of Medical Sciences and Peking Inion Medical college for technical assistance.

\section{Funding}

This study was supported by the National Natural Science Foundation of China (grant no. 81970417).

\section{Availability of data and materials}

The datasets used and/or analyzed during the current study are available from the corresponding author on reasonable request.

\section{Authors' contributions}

BTM and CWL conceived and designed the study. BTM, XBW, RZ, and SN performed the experiments. BTM, XBW and $\mathrm{QH}$ interpreted the results, analyzed the data and wrote the paper. LN, XD and ZHR analyzed the data and designed the figures and table. $\mathrm{CWL}$ and $\mathrm{QH}$ reviewed and edited the manuscript. BTM and CWL confirm the authenticity of all the raw data. All authors read and approved the final manuscript.

\section{Ethics approval and consent to participate}

Not applicable.

\section{Patient consent for publication}

Not applicable.

\section{Competing interests}

The authors declare that they have no competing interests

\section{References}

1. Messner B and Bernhard D: Smoking and cardiovascular disease: Mechanisms of endothelial dysfunction and early atherogenesis. Arterioscler Thromb Vasc Biol 34: 509-515, 2014 .
2. Siasos G, Tsigkou V, Kokkou E, Oikonomou E, Vavuranakis M, Vlachopoulos C, Verveniotis A, Limperi M, Genimata V, Papavassiliou AG, et al: Smoking and atherosclerosis: Mechanisms of disease and new therapeutic approaches. Curr Med Chem 21: 3936-3948, 2014.

3. Libby P, Buring JE, Badimon L, Hansson GK, Deanfield J, Bittencourt MS, Tokgözoğlu L and Lewis EF: Atherosclerosis. Nat Rev Dis Primers 5: 56, 2019.

4. Craig WY, Palomaki GE and Haddow JE: Cigarette smoking and serum lipid and lipoprotein concentrations: An analysis of published data. BMJ 298: 784-788, 1989.

5. Maeda K, Noguchi Y and Fukui T: The effects of cessation from cigarette smoking on the lipid and lipoprotein profiles: A meta-analysis. Prev Med 37: 283-290, 2003.

6. Selya AS and Hesse ND: Time to first cigarette and serum cholesterol levels. Soc Sci Med 174: 213-219, 2017.

7. Latha MS, Vijayammal PL and Kurup PA: Effect of exposure of rats to cigarette smoke on the metabolism of lipids. Atherosclerosis 70: 225-231, 1988.

8. Lietz M, Berges A, Lebrun S, Meurrens K, Steffen Y, Stolle K, Schueller J, Boue S, Vuillaume G, Vanscheeuwijck P, et al: Cigarette-smoke-induced atherogenic lipid profiles in plasma and vascular tissue of apolipoprotein E-deficient mice are attenuated by smoking cessation. Atherosclerosis 229: 86-93, 2013.

9. Zong C, Song G, Yao S, Guo S, Yu Y, Yang N, Guo Z and Qin S: Cigarette smoke exposure impairs reverse cholesterol transport which can be minimized by treatment of hydrogen-saturated saline. Lipids Health Dis 14: 159, 2015.

10. Ma B, Chen Y, Wang X, Zhang R, Niu S, Ni L, Di X, Han Q and Liu C: Cigarette smoke exposure impairs lipid metabolism by decreasing low-density lipoprotein receptor expression in hepatocytes. Lipids Health Dis 19: 88, 2020.

11. Lusis AJ: Atherosclerosis. Nature 407: 233-241, 2000.

12. Poirier S, Mayer G, Poupon V, McPherson PS, Desjardins R, Ly K, Asselin MC, Day R, Duclos FJ, Witmer M, et al: Dissection of the endogenous cellular pathways of PCSK9-induced low density lipoprotein receptor degradation: Evidence for an intracellular route. J Biol Chem 284: 28856-28864, 2009.

13. Urban D, Pöss J, Böhm M and Laufs U: Targeting the proprotein convertase subtilisin/kexin type 9 for the treatment of dyslipidemia and atherosclerosis. J Am Coll Cardiol 62: 1401-1408, 2013.

14. Cohen JC, Boerwinkle E, Mosley TH Jr and Hobbs HH: Sequence variations in PCSK9, low LDL, and protection against coronary heart disease. N Engl J Med 354: 1264-1272, 2006.

15. Kathiresan S and Myocardial Infarction Genetics C; Myocardial Infarction Genetics Consortium: A PCSK9 missense variant associated with a reduced risk of early-onset myocardial infarction. N Engl J Med 358: 2299-2300, 2008.

16. Sabatine MS: PCSK9 inhibitors: Clinical evidence and implementation. Nat Rev Cardiol 16: 155-165, 2019.

17. Tang ZH, Li TH, Peng J, Zheng J, Li TT, Liu LS, Jiang ZS and Zheng XL: PCSK9: A novel inflammation modulator in atherosclerosis? J Cell Physiol 234: 2345-2355, 2019.

18. Cipolla-Neto J and Amaral FGD: Melatonin as a hormone: New physiological and clinical insights. Endocr Rev 39: 990-1028, 2018.

19. Mayo JC, Sainz RM, Gonzalez Menendez P, Cepas V, Tan DX and Reiter RJ: Melatonin and sirtuins: A 'not-so unexpected' relationship. J Pineal Res 62: e12391, 2017.

20. Arioz BI, Tastan B, Tarakcioglu E, Tufekci KU, Olcum M, Ersoy N, Bagriyanik A, Genc K and Genc S: Melatonin attenuates LPS-induced acute depressive-like behaviors and microglial NLRP3 inflammasome activation through the SIRT1/Nrf2 pathway. Front Immunol 10: 1511, 2019.

21. Hardeland R: Melatonin and inflammation - Story of a double-edged blade. J Pineal Res 65: e12525, 2018.

22. Livak KJ and Schmittgen TD: Analysis of relative gene expression data using real-time quantitative PCR and the 2(-Delta Delta C(T)) Method. Methods 25: 402-408, 2001.

23. Shi J, Bai Y, Qiu S, Li Y, Kou C, Tao Y, Zhen Q, Gu Y, Yu Y, Zhang K, et al: Classified status of smoking and quitting has different associations with dyslipidemia in residents in northeast China. Clin Chim Acta 486: 209-213, 2018.

24. Muscat JE, Harris RE, Haley NJ and Wynder EL: Cigarette smoking and plasma cholesterol. Am Heart J 121: 141-147, 1991.

25. Kuzuya M, Ando F, Iguchi A and Shimokata H: Effect of smoking habit on age-related changes in serum lipids: A cross-sectional and longitudinal analysis in a large Japanese cohort. Atherosclerosis 185: 183-190, 2006. 
26. Merianos AL,JandarovRA,Khoury JC and Mahabee-Gittens EM: Tobacco smoke exposure association with lipid profiles and adiposity among U.S. adolescents. J Adolesc Health 62: 463-470, 2018.

27. Han SG, Howatt DA, Daugherty A and Gairola CG: Atherogenic and pulmonary responses of ApoE- and LDL receptor-deficient mice to sidestream cigarette smoke. Toxicology 299: 133-138, 2012.

28. Ridker PM: LDL cholesterol: Controversies and future therapeutic directions. Lancet 384: 607-617, 2014.

29. Seidah NG and Prat A: The proprotein convertases are potential targets in the treatment of dyslipidemia. J Mol Med (Berl) 85: 685-696, 2007.

30. Seidah NG, Awan Z, Chrétien M and Mbikay M: PCSK9: A key modulator of cardiovascular health. Circ Res 114: 1022-1036, 2014.

31. Park SW, Moon YA and Horton JD: Post-transcriptional regulation of low density lipoprotein receptor protein by proprotein convertase subtilisin/kexin type 9a in mouse liver. J Biol Chem 279: 50630-50638, 2004.

32. Walley KR, Thain KR, Russell JA, Reilly MP, Meyer NJ, Ferguson JF, Christie JD, Nakada TA, Fjell CD, Thair SA, et al: PCSK9 is a critical regulator of the innate immune response and septic shock outcome. Sci Transl Med 6: 258ra143, 2014.

33. Ding Z, Liu S, Wang X, Deng X, Fan Y, Shahanawaz J, Shmookler Reis RJ, Varughese KI, Sawamura T and Mehta JL: Cross-talk between LOX-1 and PCSK9 in vascular tissues. Cardiovasc Res 107: 556-567, 2015.

34. Wang X, Bian Y, Zhang R, Liu X, Ni L, Ma B, Zeng R, Zhao Z Song $X$ and Liu C: Melatonin alleviates cigarette smoke-induced endothelial cell pyroptosis through inhibiting ROS/NLRP3 axis. Biochem Biophys Res Commun 519: 402-408, 2019.

35. Ding Z, Liu S, Wang X, Deng X, Fan Y, Sun C, Wang Y and Mehta JL: Hemodynamic shear stress via ROS modulates PCSK9 expression in human vascular endothelial and smooth muscle cells and along the mouse aorta. Antioxid Redox Signal 22: $760-771,2015$.

36. Mitchell JP and Carmody RJ: NF- $\mathrm{KB}$ and the transcriptional control of inflammation. Int Rev Cell Mol Biol 335: 41-84, 2018.

37. Ahn KS and Aggarwal BB: Transcription factor NF-kappaB: A sensor for smoke and stress signals. Ann N Y Acad Sci 1056 218-233, 2005

38. Ben J, Jiang B, Wang D, Liu Q, Zhang Y, Qi Y, Tong X, Chen L, Liu X, Zhang Y, et al: Major vault protein suppresses obesity and atherosclerosis through inhibiting IKK-NF- $\kappa \mathrm{B}$ signaling mediated inflammation. Nat Commun 10: 1801, 2019.

39. Wu Y, Wang F, Fan L, Zhang W, Wang T, Du Y and Bai X: Baicalin alleviates atherosclerosis by relieving oxidative stress and inflammatory responses via inactivating the NF- $\kappa \mathrm{B}$ and p38 MAPK signaling pathways. Biomed Pharmacother 97 $1673-1679,2018$
40. Wang Z, Liu B, Zhu J, Wang D and Wang Y: Nicotine-mediated autophagy of vascular smooth muscle cell accelerates atherosclerosis via $\mathrm{nAChRs/ROS/NF- \kappa B}$ signaling pathway. Atherosclerosis 284: 1-10, 2019.

41. Liu S, Deng X, Zhang P, Wang X, Fan Y, Zhou S, Mu S, Mehta JL and Ding Z: Blood flow patterns regulate PCSK9 secretion via MyD88-mediated pro-inflammatory cytokines. Cardiovasc Res 116: 1721-1732, 2020

42. Morgan MJ and Liu ZG: Crosstalk of reactive oxygen species and NF- $\kappa$ B signaling. Cell Res 21: 103-115, 2011.

43. Loloei S, Sepidarkish M, Heydarian A, Tahvilian N, Khazdouz M, Heshmati J and Pouraram H: The effect of melatonin supplementation on lipid profile and anthropometric indices: A systematic review and meta-analysis of clinical trials. Diabetes Metab Syndr 13: 1901-1910, 2019.

44. Agil A, Navarro-Alarcón M, Ruiz R, Abuhamadah S, El-Mir MY and Vázquez GF: Beneficial effects of melatonin on obesity and lipid profile in young Zucker diabetic fatty rats. J Pineal Res 50: 207-212, 2011.

45. Hussain SA: Effect of melatonin on cholesterol absorption in rats. J Pineal Res 42: 267-271, 2007.

46. Hoyos M, Guerrero JM, Perez-Cano R, Olivan J, Fabiani F, Garcia-Pergañeda A and Osuna C: Serum cholesterol and lipid peroxidation are decreased by melatonin in diet-induced hypercholesterolemic rats. J Pineal Res 28: 150-155, 2000.

47. Yin J, Li Y, Han H, Chen S, Gao J, Liu G, Wu X, Deng J, Yu Q, Huang X, et al: Melatonin reprogramming of gut microbiota improves lipid dysmetabolism in high-fat diet-fed mice. J Pineal Res 65: e12524, 2018.

48. Kauppinen A, Suuronen T, Ojala J, Kaarniranta K and Salminen A: Antagonistic crosstalk between NF-кB and SIRT1 in the regulation of inflammation and metabolic disorders. Cell Signal 25: 1939-1948, 2013.

49. Xu F, Xu J, Xiong X and Deng Y: Salidroside inhibits MAPK, $\mathrm{NF}-\kappa \mathrm{B}$, and STAT3 pathways in psoriasis-associated oxidative stress via SIRT1 activation. Redox Rep 24: 70-74, 2019.

50. Singh CK, Chhabra G, Ndiaye MA, Garcia-Peterson LM, Mack NJ and Ahmad N: The role of sirtuins in antioxidant and redox signaling. Antioxid Redox Signal 28: 643-661, 2018.

This work is licensed under a Creative Commons Attribution-NonCommercial-NoDerivatives 4.0 International (CC BY-NC-ND 4.0) License. 